\section{\begin{tabular}{|l|}
\hline Doxorubicin affecte \\
\hline ATP synthesis \\
Cytoskeleton \\
Cytoskeleton regulation \\
Glycolysis \\
Protein folding \\
Redox regulation \\
TCA cycle \\
\hline
\end{tabular}}

Reversible by quercetin pretreatment
Cell cycle
Collagen formation
DNA repair
DNA synthesis
Growth regulation
Hemostasis
Ion transport
Nuclear assembly
Protein degradation
Signal transduction
Translational control

No relevant to quercetin pretreatment

\title{
Quercetin-induced cardioprotection against doxorubicin cytotoxicity
}

Chen et al. 


\title{
Quercetin-induced cardioprotection against doxorubicin cytotoxicity
}

\author{
Jing-Yi Chen, Ren-Yu Hu and Hsiu-Chuan Chou*
}

\begin{abstract}
Background: Cancer has continually been the leading cause of death worldwide for decades. Thus, scientists have actively devoted themselves to studying cancer therapeutics. Doxorubicin is an efficient drug used in cancer therapy, but also produces reactive oxygen species (ROS) that induce severe cytotoxicity against heart cells. Quercetin, a plant-derived flavonoid, has been proven to contain potent antioxidant and anti-inflammatory properties. Thus, this in vitro study investigated whether quercetin can decrease doxorubicin-induced cytotoxicity and promote cell repair systems in cardiomyocyte H9C2 cells.

Results: Proteomic analysis and a cell biology assay were performed to investigate the quercetin-induced responses. Our data demonstrated that quercetin treatment protects the cardiomyocytes in a doxorubicin-induced heart damage model. Quercetin significantly facilitated cell survival by inhibiting cell apoptosis and maintaining cell morphology by rearranging the cytoskeleton. Additionally, 2D-DIGE combined with MALDI-TOF MS analysis indicated that quercetin might stimulate cardiomyocytes to repair damage after treating doxorubicin by modulating metabolic activation, protein folding and cytoskeleton rearrangement.

Conclusion: Based on a review of the literature, this study is the first to report detailed protective mechanisms for the action of quercetin against doxorubicin-induced cardiomyocyte toxicity based on in-depth cell biology and proteomic analysis.
\end{abstract}

Keywords: Quercetin, Doxorubicin, Proteomics, DIGE, MALDI-TOF, Cardiomyocytes

\section{Background}

Doxorubicin is a chemotherapy drug, commonly used in various cancer treatments, such as breast cancer, lung cancer and several other carcinoma types [1-3]. The principal mechanism of doxorubicin is chelating DNA, inhibiting topoisomerase II and then producing free radicals to kill cancer cells. Reported side effects of doxorubicin include cardiotoxicity, comprising cardiomyopathy and ultimately fatal congestive heart failure. Because myocardia are particularly sensitive to reactive oxygen species (ROS), cumulative doxorubicin in vivo causes irreversible damage to heart cells, thus restricting clinical use of this drug [4]. Although the specific causal mechanism of doxorubicininduced cardiotoxicity remains largely unclear, most of the evidence has indicated that doxorubicin is reduced to its semiquinone form by a mitochondria electron transport system. The semiquinone subsequently reacts with

\footnotetext{
* Correspondence: chouhc@mail.nhcue.edu.tw

Department of Applied Science, National Hsinchu University of Education, Hsinchu, Taiwan
}

oxygen, iron, and hydrogen peroxide to produce ROS causing cell apoptosis and myocyte damage [5,6]. In addition, global analysis of doxorubicin-induced cellular oxidative stress has indicated that doxorubicin treatment contributes to the over-expression of anti-oxidant proteins such as glutathione reductase and peroxiredoxin in brain cells, lung cells and heart cells [7-9].

Quercetin, a type of polyphenolic compound found in various plant products, possesses anti-oxidant, antiproliferative, anti-inflammatory and anti-histamine properties. Several reports have indicated that quercetin exerts protective effects on various cells, including myocytes, testes, renal cells and liver cells in ischemia and reperfusion injury [10]. A study conducted in 1992 determined that quercetin reduces the oxidative stress caused by ischemia and reperfusion in cardiomyocytes by inhibiting the xanthine dehydrogenase and xanthine oxidase system [11]. Several reports have also indicated that quercetin and isorhamnetin scavenge ROS and inhibit the activation of ERK and MAP kinase in ROS-induced cardiomyopathy $[12,13]$. In 
cancer therapy, combining quercetin with doxorubicin augmented the effects of doxorubicin in highly invasive breast cancer cells [14] and can protect cardiomyocytes from doxorubicin-induced toxicity by chelating iron, inducing antioxidant activity, and inhibiting carbonyl reductase [15]. Regarding proteomic analysis, the results also indicated that quercetin could down-regulate Ras GTPase-activating-like proteins and heat shock protein-90 to reduce cell migratory ability and cell survival, respectively, in malignant cancers [16,17]. Although quercetin has been reported to play a role in protecting myocardial cells from ischemia and reperfusion injury, its protective mechanism remains unclear.

To investigate the role of quercetin in alleviating doxorubicin-induced cardiotoxicity, we examined the protective ability of quercetin in doxorubicin-treated rat cardiomyocytes by performing cell biological assays, such as cell viability and apoptotic analysis, as well as a quantitative proteomic analysis based on 2D-DIGE and MALDI-TOF MS identification [18].

\section{Methods}

\section{Chemicals and reagents}

Generic chemicals were purchased from Sigma-Aldrich (St. Louis, USA), while reagents for 2D-DIGE were purchased from GE Healthcare (Uppsala, Sweden). All primary antibodies were purchased from Genetex (Hsinchu, Taiwan) and anti-mouse, and anti-rabbit secondary antibodies were purchased from GE Healthcare (Uppsala, Sweden). All the chemicals and biochemicals used in this study were of analytical grade.

\section{Cell lines and cell culture}

The rat cardiomyocyte cell line H9C2 was purchased from American Type Culture Collection (ATCC) (Manassas, VA) and was maintained in Dulbecco's modified Eagle's medium (DMEM) supplemented with $10 \%$ (v/v) FCS, L-glutamine $(2 \mathrm{mM})$, streptomycin $(100 \mu \mathrm{g} / \mathrm{mL})$ and penicillin (100 IU/mL) (all from Gibco-Invitrogen Corp., UK). Cells were incubated in a humidified incubator at $37^{\circ} \mathrm{C}$ and $5 \% \mathrm{CO}_{2}$. and passaged at $80-90 \%$ confluence by trypsinization according to standard procedures.

\section{MTT cell viability assay}

The detailed MTT experimental procedure has been described in our previous study [19].

\section{Immunofluorescence}

Cells were plated onto coverslips (VWR international) for overnight incubation and subsequently fixed with PBS containing 4\% (v/v) paraformaldehyde for $25 \mathrm{~min}$. After washing three times in PBS, samples were permeabilized in PBS containing $0.2 \%(\mathrm{v} / \mathrm{v})$ Triton X-100 for $10 \mathrm{~min}$. and then rinsed and blocked in PBS containing 5\% (w/v) BSA for $10 \mathrm{~min}$ prior to incubate with primary antibodies diluted in 2.5\% BSA/PBS for $1 \mathrm{~h}$. After PBS washings, samples were incubated with the appropriate fluorescently labeled secondary antibodies diluted in $2.5 \%$ BSA/PBS for $1 \mathrm{~h}$. Samples were then washed three times with $\mathrm{PBS}$ and briefly rinsed with $\mathrm{dd}_{2} \mathrm{O}$ twice before applying to Vectashield mounting medium (Vector Lab). Coverslip edges were sealed with nail polish onto glass slides $(\mathrm{BDH})$ and then air-dried in the dark at $4^{\circ} \mathrm{C}$. For image analysis, cells were visualized using a Zeiss Axiovert Z1 fluorescent microscope (Carl Zeiss Inc., Germany). Identical laser intensities were used to detect the same immunostained proteins to obtain non-saturated images. Images were exported as .tif files using the Zeiss Axioversion 4.0.

\section{Flow cytometry analysis for apoptosis detection}

Annexin-V/propidium iodide (PI) double assay was performed using the Annexin V, Alexa Fluor ${ }^{\oplus} 488$ Conjugate Detection kit (Life technologies). Following doxorubicin treatment, cells were typsinized from culture dish and washed twice with cold PBS. $1 \times 10^{6}$ cells were resuspended in $500 \mu \mathrm{L}$ binding buffer and stained with $5 \mu \mathrm{L}$ Alexa Fluor 488 conjugated annexin $\mathrm{V}$ according to the manufacturer's instructions. $1 \mu \mathrm{L} 100 \mu \mathrm{g} / \mathrm{mL}$ propidium iodide (PI) was mixed gently to cells for $15 \mathrm{~min}$ at room temperature in the dark. After incubation period, samples were subjected to FCM analysis in $1 \mathrm{~h}$. using BD Accuri C6 Flow Cytometry (BD Biosciences, San Jose, CA). The data were analyzed using Accuri CFlow ${ }^{\circledR}$ and CFlow Plus analysis software (BD Biosciences).

\section{Immunoblotting analysis}

Immunoblotting analysis was used to validate the differential abundance of mass spectrometry identified proteins. The detailed experimental procedures were described in

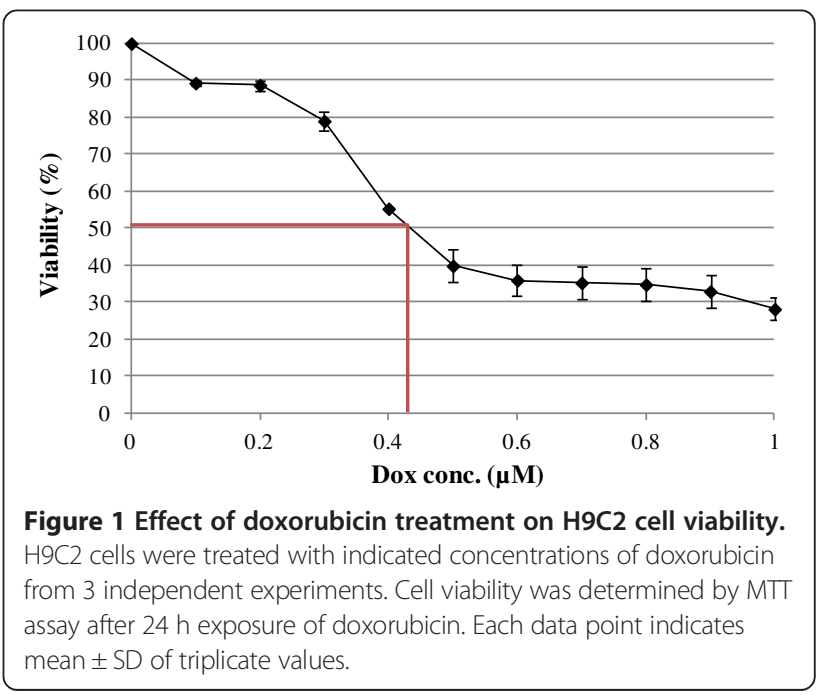




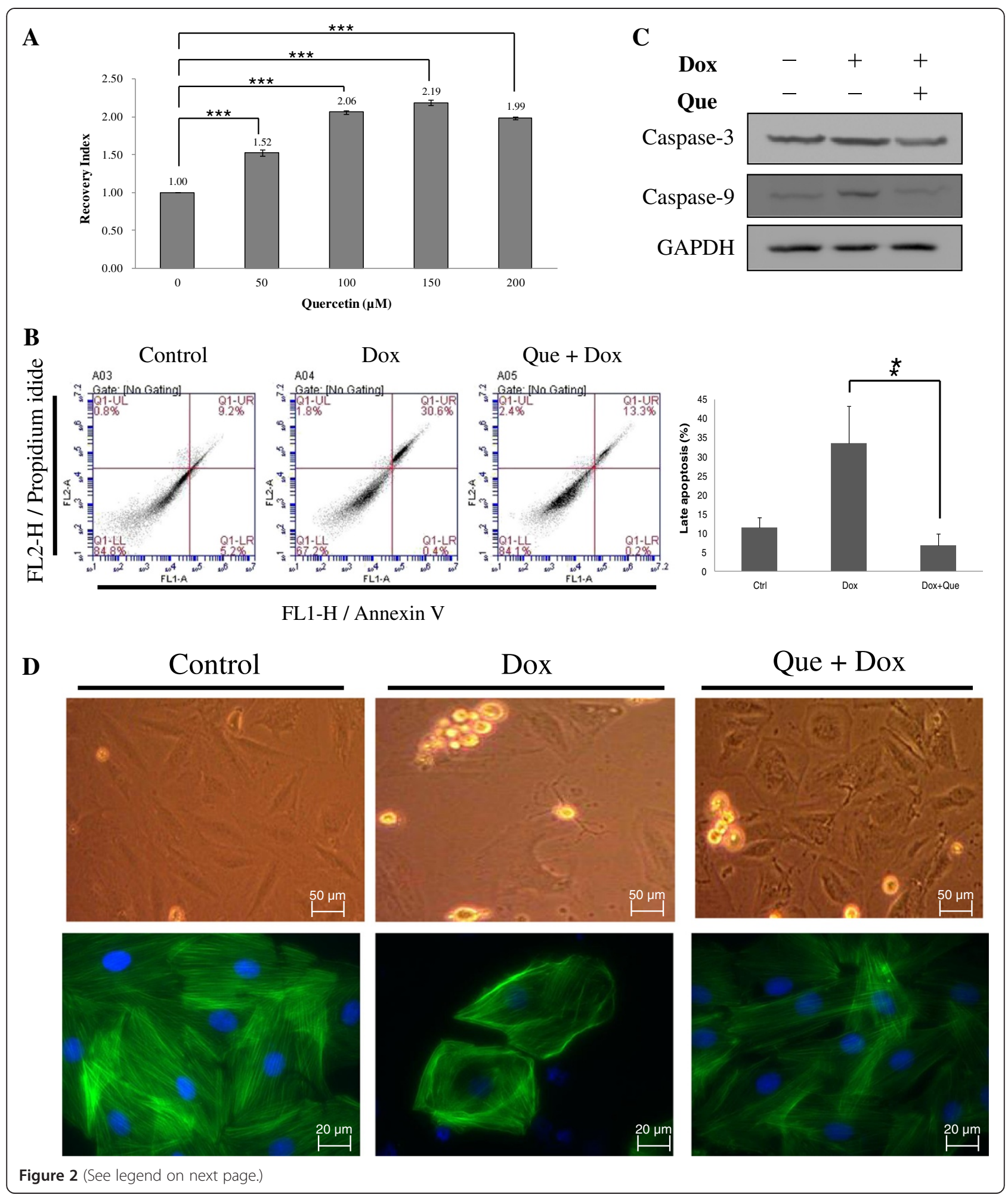


(See figure on previous page.)

Figure 2 Effects of quercetin on doxorubicin-induced changes of cell viability, cell apoptosis and cell morphology in H9C2 cells. (A) MTT-based viability assays were performed on H9C2 cell cultures following treatments with different concentrations of quercetin $(50 \mu \mathrm{M}$, $100 \mu \mathrm{M}$, $150 \mu \mathrm{M}$ and $200 \mu \mathrm{M}$ ) or left untreated. Values were normalized against untreated samples and were the average of 4 independent measurements +/- the standard deviation. The statistic analysis was performed with two group paired Student t-test. (B) Typical dot plot diagrams detected annexin V-FITC and PI staining represent untreated, doxorubicin-treated, and quercetin-pretreated followed by doxorubicin-treated cells. The $x$-axis and $y$-axis stand for the intensity of annexin V-FITC and PI, respectively. The lower left area of presented background staining by annexin V-FITC and PI in normal cells, and apoptotic signals located in the right area. This figure is representative of 4 replicates. The statistic analysis of the replicates was listed in right panel. (C) The levels of caspase 3 and caspase 9 in H9C2 cells were detected by immunoblotting. GAPDH served as a sample loading control. (D) Cell morphology and protein location of F-actin in H9C2 cells were analyzed by immunostaining. H9C2 cells on coverslips were either left untreated, treated with doxorubicin or pre-treated with quercetin prior to doxorubicin treatment before fixation and staining. F-actin was stained with phalloidin and nuclei were stained with DAPI. Each set of five fields were taken using the same exposure and images are representative of five different fields. In (B) (D), H9C2 cells were untreated, $0.45 \mu \mathrm{M}$ of doxorubicin for $24 \mathrm{~h}$, or $100 \mu \mathrm{M}$ of quercetin for $4 \mathrm{~h}$ followed by $0.45 \mu \mathrm{M}$ of doxorubicin for $24 \mathrm{~h}$.

our previous reports [20-22]. All primary antibodies used for expression validation were purchased from Genetex (Hsinchu, Taiwan).

\section{D-DIGE, gel image analysis, protein staining, in-gel digestion and MALDI-TOF MS analysis}

The detailed experimental procedures have been described in our previous publications [23-25]. Notably, peaks in the mass range of $m / z 800$-3000 were used to generate a peptide mass fingerprint that was searched against the Swiss-Prot/TrEMBL database (released on August 2011) with 531473 entries using Mascot software v2.3.02 (Matrix Science, London, UK). The parameters used for Mascot search are listed: mouse; tryptic digest with a maximum of 1 missed cleavage; carbamidomethylation of cysteine, partial protein N-terminal acetylation, partial methionine oxidation and partial modification of glutamine to pyroglutamate and a mass tolerance of $50 \mathrm{ppm}$. Identification was accepted based on significant MASCOT Mowse scores $(p<0.05)$, spectrum annotation and observed versus expected molecular weight and $\mathrm{p} I$ on $2-\mathrm{DE}$ as well as at least 5 peptides in each identified protein.

\section{Results}

Quercetin facilitates cell survival and maintains cell morphology in doxorubicin-induced cell death in $\mathrm{H} 9 \mathrm{C} 2$ cells To evaluate the effect of doxorubicin on rat cardiomyocytes ( $\mathrm{H} 9 \mathrm{C} 2)$, we exposed the cells to doxorubicin in a range of $0-1 \mu \mathrm{M}$ for $24 \mathrm{~h}$ in a serum-free medium. After exposure to doxorubicin, dose dependent loss of cell viabilities was observed in the $\mathrm{H} 9 \mathrm{C} 2$ cells in 3 independent experiments using MTT assays (Figure 1). At a concentration of $0.45 \mu \mathrm{M}$, a significant loss $(50 \%)$ of cell viability was detected after $24 \mathrm{~h}$. To verify the role of quercetin regarding the recovery of doxorubicin-induced cardiomyopathy, we investigated the changes in cell viability in the H9C2 cells incubated in $0 \mu \mathrm{M}, 50 \mu \mathrm{M}, 100 \mu \mathrm{M}$, $150 \mu \mathrm{M}$ and $200 \mu \mathrm{M}$ quercetin for $4 \mathrm{~h}$, followed by 24 h-exposure to $0.45 \mu \mathrm{M}$ doxorubicin. Our results demonstrated that cell viability was significantly improved using quercetin in concentrations from 50 to $200 \mu \mathrm{M}$ (Figure 2A).

Because excess ROS stress from doxorubicin-treated cardiomyocyte alters redox homeostasis and induces cell death, cell apoptosis was further detected using FACS. During cell apoptosis, phosphatidylserine is translocated to the outer surface of the plasma membrane, which has a high affinity to annexin V-FITC, and PI can penetrate the cell nucleus. As shown in Figure 2B, the apoptotic rate increased from $4.9 \%$ to $61.4 \%$ after doxorubicin treatment, whereas the apoptotic rate decreased to $9.5 \%$ after the $\mathrm{H} 9 \mathrm{C} 2$ cells were pretreated with quercetin before doxorubicin treatment. In addition, the levels of the proteolytic enzymes, caspase 9 and caspase 3, were detected using immunoblotting in control, doxorubicin-treated and quercetin-pretreated $\mathrm{H} 9 \mathrm{C} 2$ cells. Figure $2 \mathrm{C}$ indicates increased expression levels of the apoptosis factors for caspase 3 and caspase 9 after doxorubicin treatment. Quercetin protected the H9C2 cells from doxorubicininduced cell injury by inhibiting the expressions of caspase 3 and caspase 9. Additionally, immunostained images of F-actin indicated that doxorubicin treatment affected cytoskeletal protein reorganization, causing cell morphology alternation (Figure 2D). Therefore, quercetin pretreatment is essential to maintaining doxorubicin-induced morphological changes.

\section{D-DIGE analysis of untreated and doxorubicin-treated H9C2 cells and quercetin pretreatment followed by doxorubicin treatment}

To fully understand the roles of doxorubicin and quercetin pretreatment in $\mathrm{H} 9 \mathrm{C} 2$ cells, lysates of cells untreated, treated with doxorubicin, or treated with doxorubicin after pretreatment of quercetin, were subjected to 2D-DIGE analysis. The results of the 2D-DIGE analysis and DeCyder processing identified 2156 protein spots, and 73 proteins exhibited differential expression $(\geqq 1.5$ fold or $\leqq-1.5$ fold; $p<0.05$ ) among the 3 conditions (Figure 3). Additional file 1: Table S1 shows the 73 proteins that were identified using MALDI-TOF MS, and 31 of the 73 identified 


A
\begin{tabular}{|c|c|c|c|}
\hline & Cy2 $(100 \mu \mathrm{g})$ & Cy3 $(100 \mu \mathrm{g})$ & Cy5 $(100 \mu \mathrm{g})$ \\
\hline Gel 1 & pool & Ctrl & Dox \\
\hline Gel 2 & pool & Dox & Dox+Que \\
\hline Gel 3 & pool & Dox+Que & Ctrl \\
\hline Gel 4 & pool & Ctrl & Dox+Que \\
\hline Gel 5 & pool & Dox+Que & Dox \\
\hline
\end{tabular}

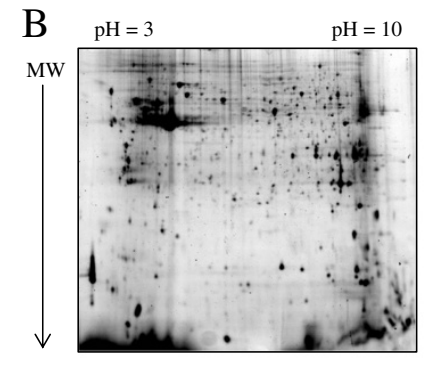

Control

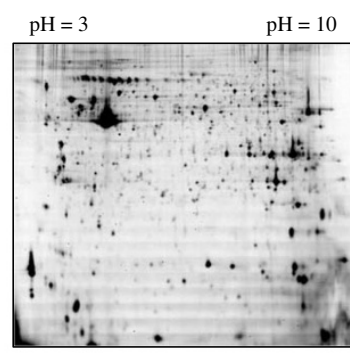

Dox

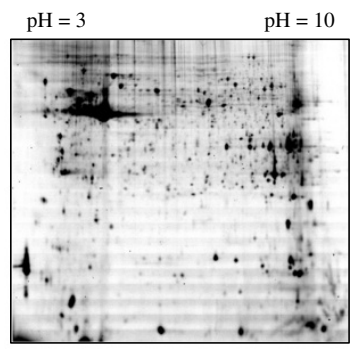

Que + Dox

C

$$
\downarrow
$$

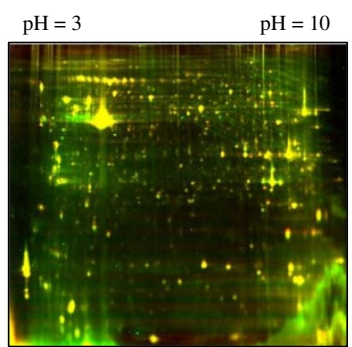

Green: Control Red: Dox

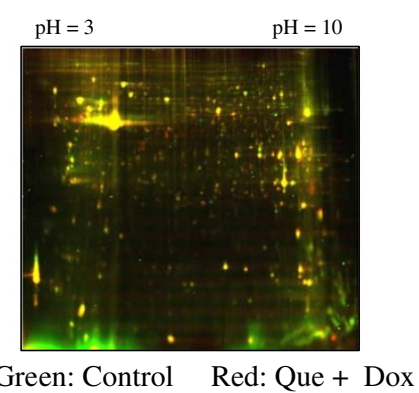

$\mathrm{D}$

$$
\mathrm{pH}=3 \quad \mathrm{pH}=10
$$

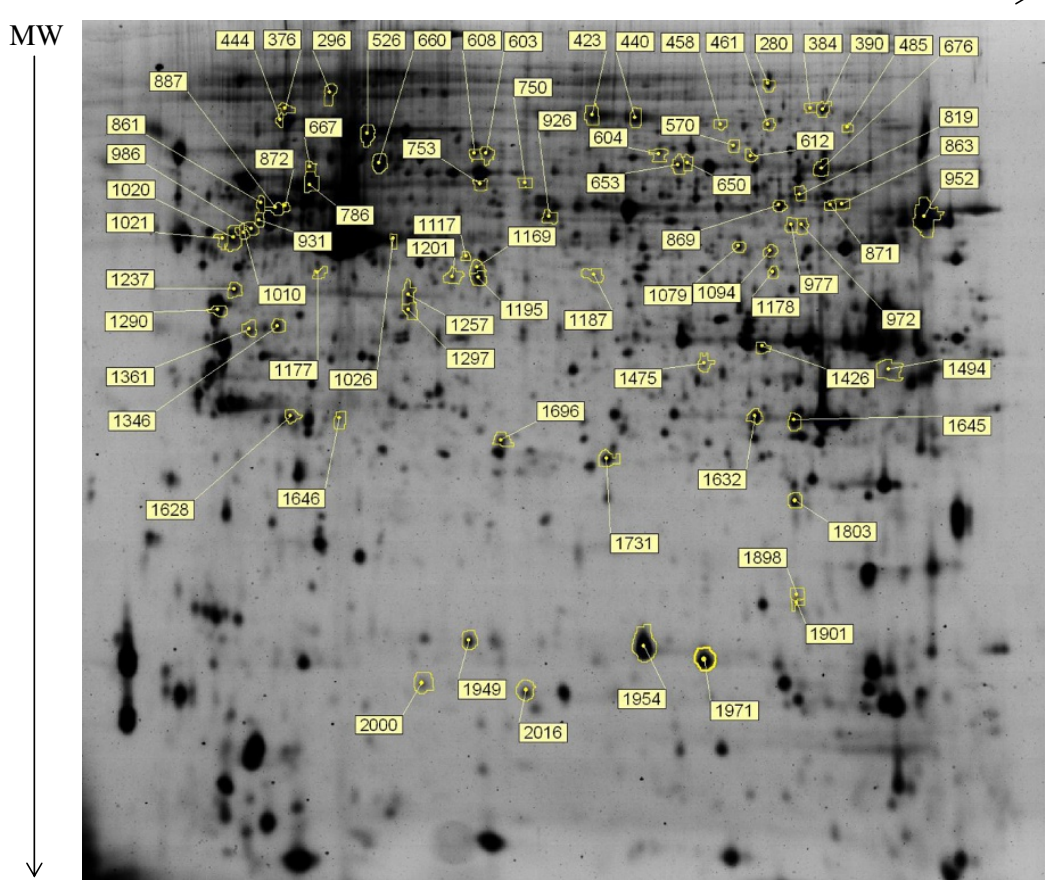

Figure $\mathbf{3}$ (See legend on next page.) 
(See figure on previous page.)

Figure 3 2D-DIGE analysis of $\mathrm{H} 9 \mathrm{C} 2$ cells in response to doxorubicin treatment and pre-treatment with quercetin. (A) Samples arrangement for a triplicate 2D-DIGE experiment. (B) Protein samples (100 $\mu \mathrm{g}$ each) were labeled with Cy-dyes and separated using $24 \mathrm{~cm}$, pH 3-10 non-linear IPG strips. 2D-DIGE images of the protein samples from $\mathrm{H} 9 \mathrm{C} 2$ cells in response to doxorubicin treatment and pre-treatment with quercetin at appropriate excitation and emission wavelengths were shown as well as overlaid pseudo-colored images processed with ImageQuant Tool (GE Healthcare) (C). (D) Protein samples (100 $\mathrm{\mu g}$ each) purified from total cell lysates were labeled with Cy-dyes and separated using $24 \mathrm{~cm}, \mathrm{pH} 3-10$ non-linear IPG strips. The differentially expressed protein features were annotated with spot numbers. In this 2D-DIGE experiment, H9C2 cells were untreated, $0.45 \mu \mathrm{M}$ of doxorubicin for $24 \mathrm{~h}$, or $100 \mu \mathrm{M}$ of quercetin for $4 \mathrm{~h}$ followed by $0.45 \mu \mathrm{M}$ of doxorubicin for $24 \mathrm{~h}$.

protein spots that displayed doxorubicin-dependent alteration can be reversed by pretreating with quercetin (Additional file 1: Table S1 and Additional file 2). For example, the $78 \mathrm{kDa}$ glucose-regulated protein (GRP-78) (No.444) was up-regulated (1.63 fold) in the doxorubicintreated cells, whereas quercetin reduced the overexpression of doxorubicin-treated GRP-78 (-1.85 fold). The result suggested that the protective mechanisms of quercetin significantly altered the levels of chaperone proteins during doxorubicin-treatment in the cardiomyocytes. Figure 4A shows the functional distribution of the identified proteins from the 2D-DIGE results. Most of the proteins identified using MALDI-TOF MS were associated with the cytoskeletal element and cell migration
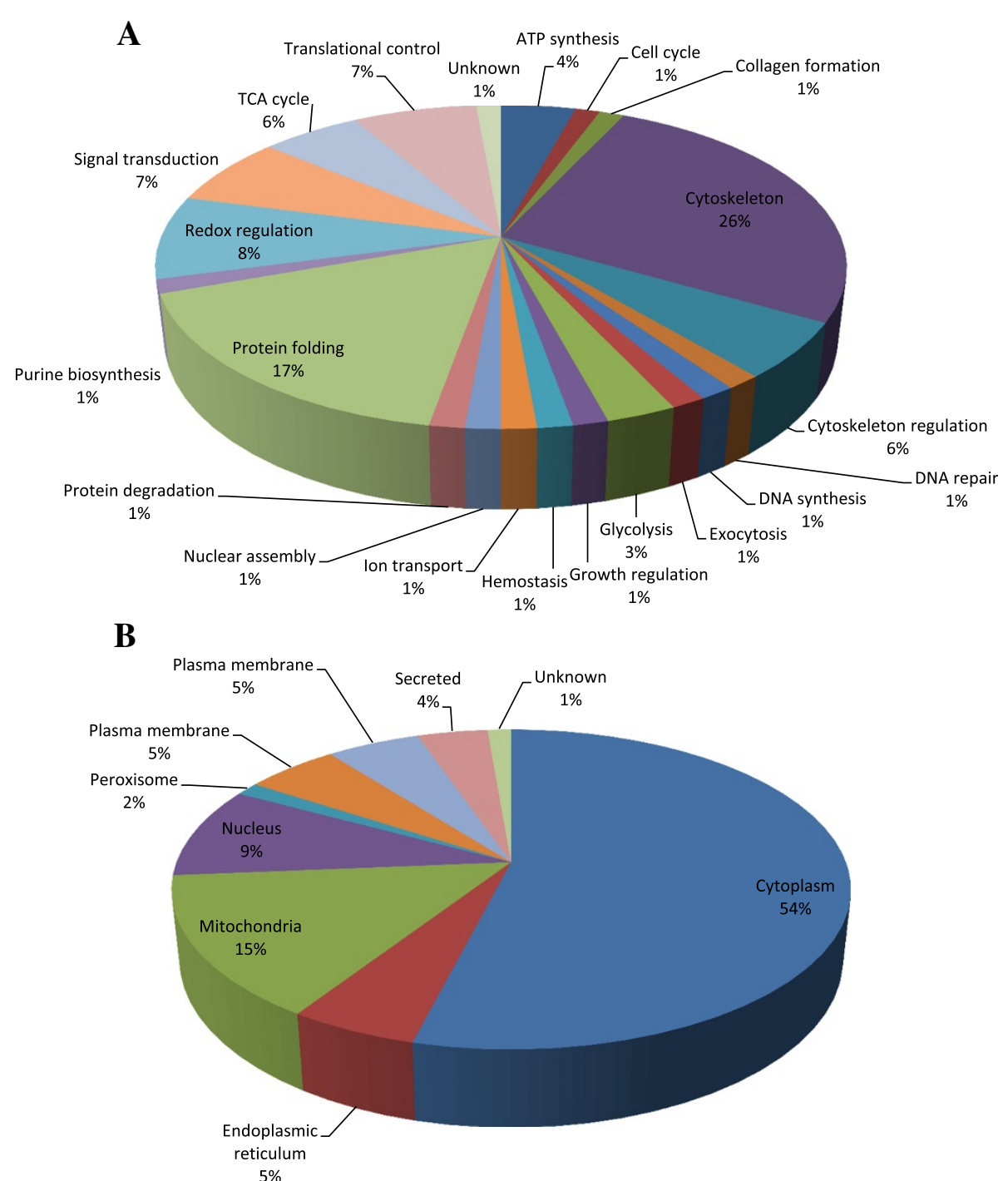

Figure 4 Percentage of total differentially expressed proteins identified by 2D-DIGE/MALDI-TOF MS for H9C2 cells in response to doxorubicin treatment and pre-treatment with quercetin according to their biological functions $(A)$ and sub-cellular locations (B). 


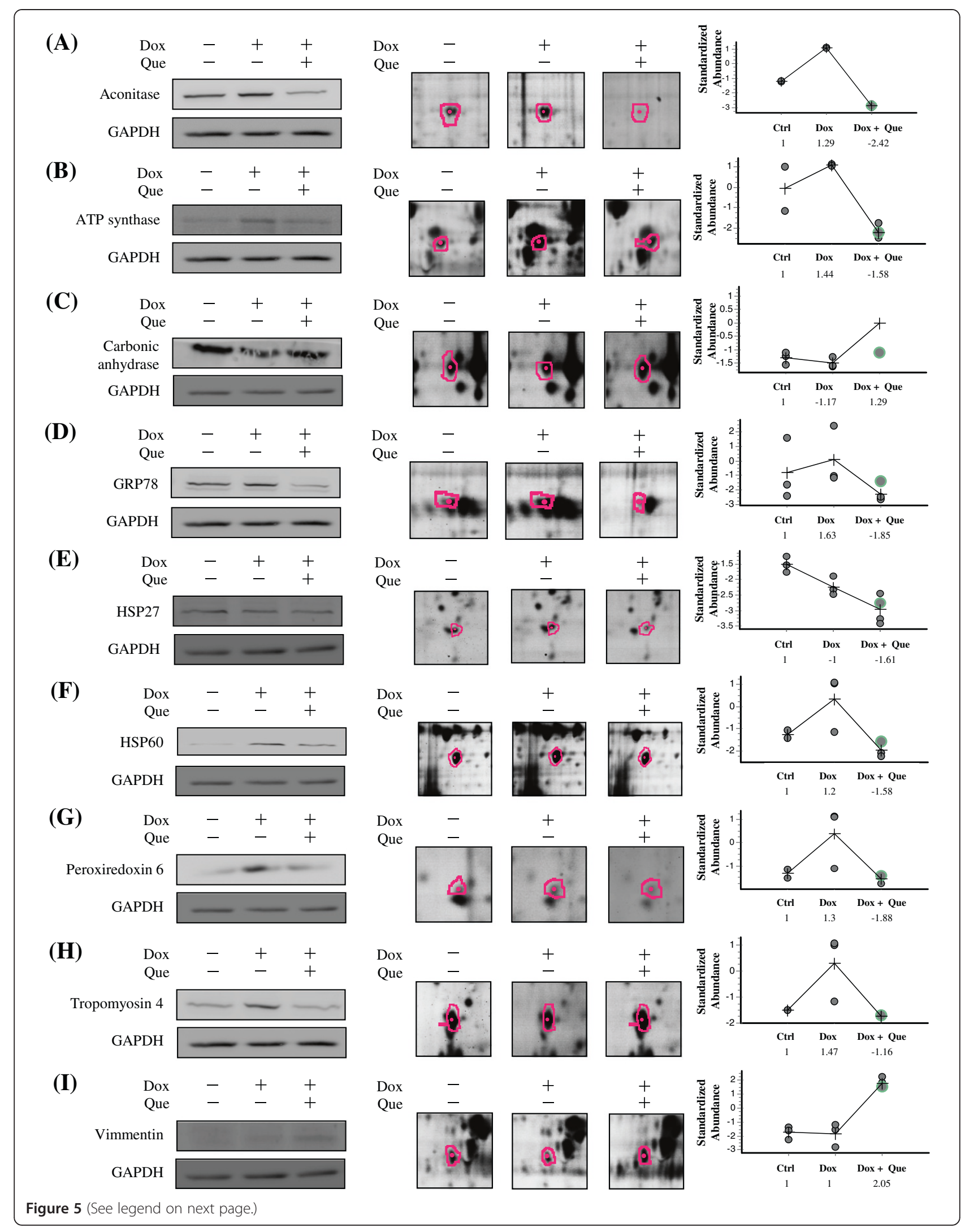


(See figure on previous page.)

Figure 5 Representative immunoblotting analyses for selected differentially expressed proteins identified by proteomic analysis of $\mathrm{H} 9 \mathrm{C} 2$ cells in response to doxorubicin treatment and pre-treatment with quercetin. The levels of identified proteins, (A) Aconitase,

(B) ATP synthase, (C) Carbonic anhydrase, (D) GRP78, (E) HSP27, (F) HSP60, (G) Peroxiredoxin 6, (H) Tropomyosin 4, (I) Vimmentin, in H9C2 cells in response to doxorubicin treatment and pre-treatment with quercetin were confirmed by immunoblot, while GAPDH was used as loading controls (left panels). The protein expression maps and two-dimensional spot images were shown in right panels and middle panels, respectively.

as well as protein biosynthesis and metabolism, implying that quercetin is crucial for sustaining cytoskeletal and metabolic alternations responding to oxidative damage in the cardiomyocytes. During doxorubicinmediated cardiomyopathy, the majority of the identified proteins were located in the cytoplasm and the nucleus (Figure 4B).

\section{Verifying the 2D-DIGE results by using immunoblotting} and immunostaining

The levels of aconitase, ATP synthase, carbonic anhydrase, GRP78, HSP27, HSP60, peroxiredoxin 6, tropomyosin 4, vimmentin and cofilin-1 were examined using immunoblotting and immunostaining to validate the results of the 2D-DIGE analysis. The results indicated that aconitase, ATP synthase, GRP78, HSP60, peroxiredoxin 6 , tropomyosin 4 and cofilin-1 were overexpressed in response to doxorubicin. However, quercetin suppressed the expression of the proteins during doxorubicin treatment in the H9C2 cells (Figure 5 and Figure 6). These results are consistent with the 2D-DIGE results.

\section{Discussion}

Myocardial damage induced by doxorubicin was primarily caused by chelating DNA, inhibiting topoisomerase II and producing free radicals. Based on these concepts, numerous studies have evaluated the effects of doxorubicininduced toxicity and the mechanisms that contribute to protecting cardiomyocytes [26-28]. In our previous studies, we reported on the cellular oxidative targets during heart damage induced by doxorubicin [29]. Additionally, we demonstrated that quercetin might dephosphorylate Src kinase activity in ROS-induced $\mathrm{H} 9 \mathrm{C} 2$ cells and block ROS-induced inflammatory responses through STAT3 kinase. These activities contribute to preventing ischemia and reperfusion injury in cardiomyocytes [30]. In this study, we determined that quercetin treatment protected cardiomyocytes in the doxorubicin-induced heart damage model. Moreover, quercetin significantly facilitated cell survival by inhibiting cell apoptosis and maintaining cell morphology by inducing cytoskeletal protein rearrangement. Furthermore, after the proteomic analysis, we observed dramatic reductions in the $\mathrm{H} 9 \mathrm{C} 2$ proteins involving protein folding, redox-regulation, and energy

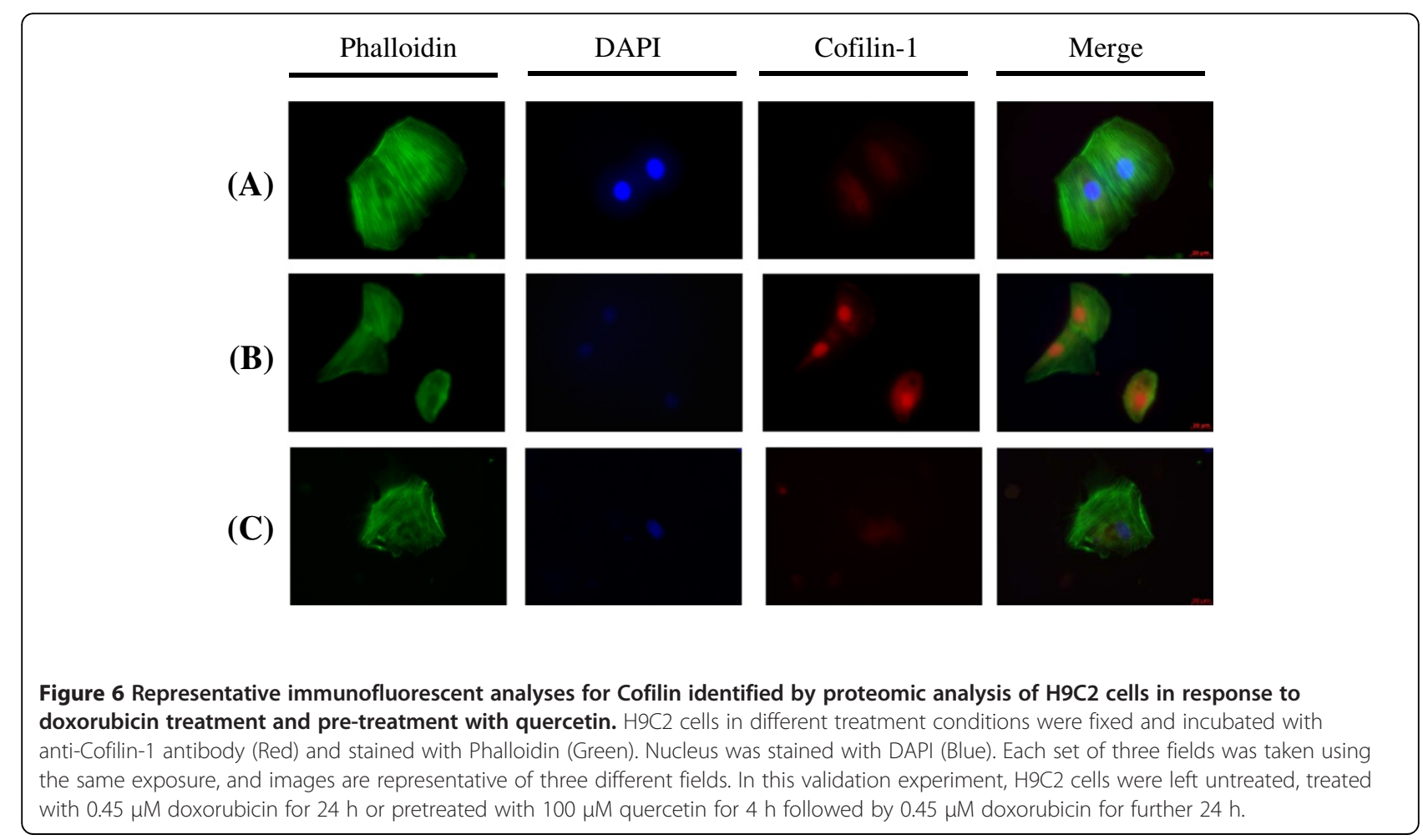


metabolism, as well as significant increases in proteins involving cytoskeleton and cytoskeleton regulatory proteins among the lysates of cells that were untreated, treated with doxorubicin, or treated with doxorubicin after pretreatment with quercetin. We suggest that cardiomyocytes develop defense mechanisms to overcome doxorubicin-induced ROS accumulation, which causes cell damage and cell death. The defense mechanisms include the overexpression of redox-modulated proteins to scavenge doxorubicin-induced ROS. In this study, pretreating with quercetin might scavenge ROS, diminishing oxidative stress and subsequently downregulating redoxregulatory proteins including glutamate dehydrogenase 1 , isocitrate dehydrogenase, NADP-dependent malic enzyme, retinal dehydrogenase 1 and peroxiredoxin-6.

Moreover, we also observed that most proteins critical for modulating protein folding were down-regulated during pretreatment with quercetin which might account for the decreased concentrations of ROS causing the decrease of incorrectly folding proteins and attenuating the expression of chaperone proteins such as $60 \mathrm{kDa}$ heat shock protein, $78 \mathrm{kDa}$ glucose-regulated protein, alpha-crystallin B, heat shock protein beta-1, Stress-induced-phosphoprotein 1 and T-complex protein 1. Similar results have been reported in ethanol- and arsenite-induced oxidative stress, which caused over-expression of anti-oxidative stress proteins. However, the over-expression was significantly alleviated by quercetin pre-treatment $[31,32]$. In addition to these studies, the quercetin-induced downregulation of the protective proteins might account for the quercetintreated cells exhibiting higher sensitivity to ROS damage, such as cancer cells $[33,34]$. Several key regulatory proteins mediated the interaction of heat shock proteins to inhibit apoptosis. The intrinsic pathway of caspase-mediated apoptosis was stimulated by c-Jun kinase, resulting in the release of cytochrome $\mathrm{c}$ from the mitochondria, and the subsequent activation of a caspase cascade involving caspase 8 and caspase 3 . They were each inhibited by heat shock cognate 71 , which interacted with Bcl-2 through Bag-1, enabling the complex to be incorporated into the mitochondrial membrane to inhibit apoptosis [35]. In this study, heat shock cognate 71 was upregulated in the quercetin-pretreated $\mathrm{H} 9 \mathrm{C} 2$ cells, implying that heat shock cognate 71 is essential for protecting $\mathrm{H} 9 \mathrm{C} 2$ cells from doxorubicin-induced apoptosis.

Quercetin was also observed to modulate the expression of cytoskeletal proteins (e.g., tubulins) and migrationregulated proteins (e.g., tubulin polymerization-promoting proteins) after encountering doxorubicin-induced damage [36]. Our immunofluorescence study demonstrated that quercetin can promote F-actin organization. Proteomic data also suggested that actin molecules were overexpressed during quercetin pretreatment, implying that quercetin causes the efficient regulation of protrusion dynamics and the wound healing of doxorubicin-damaged cardiomyocytes.

Our proteomic analysis indicated that quercetin pretreatment might down-regulate the levels of proteins involving energy metabolism including mitochondrial ATP synthesis, glycolytic proteins and TCA cycle proteins. Similar results were also reported by Dihal et al., who observed that glycolytic proteins were significantly downregulated in their report. Additionally, Shoshan et al. reported that quercetin can modulate mitochondrial energy production by interacting with ATP synthase and blocking the enzyme's activity $[37,38]$. The current proteomic analysis corresponded with these results.

Our preliminary data indicated that quercetin reduces but enhances the cytotoxicity of doxorubicin on cardiomyocyte H9C2 cells and liver cancer HepG2 cells, respectively (data not shown). This observation suggested the potential of combining quercetin and doxorubicin for treating liver cancer. Although no direct evidence indicates the cooperative effect of quercetin and doxorubicin on other cancer treatment, performing relevant evaluations of other cancers is worthwhile in the future.

In summary, this study is the first to report on the principle mechanism of quercetin against doxorubicininduced cytotoxicity in cardiomyocytes, using cell biology and a quantitative proteomic analysis. The information obtained in this study presents the potential of combining quercetin with doxorubicin to achieve reduced cardiotoxicity in cancer chemotherapy.

\section{Conclusions}

This study is the first to report detailed protective mechanisms for the action of quercetin against doxorubicininduced cardiomyocyte toxicity. Quercetin might stimulate cardiomyocytes to repair damage after treating doxorubicin by modulating metabolic activation, protein folding and cytoskeleton rearrangement.

\section{Additional files}

Additional file 1: Table S1. Differentially expressed proteins were listed alphabetically after 2D-DIGE and MALDI-TOF Mass spectrometry analysis in $\mathrm{H} 9 \mathrm{C} 2$ cells in response to doxorubicin treatment and quercetin pretreatment. The average ratios of these 73 spots are differentially expressed among untreated (control), doxorubicin-treated and quercetin-pretreated followed by doxorubicin-treated cells, calculated from triplicate gels.

Additional file 2: Raw spectra of the identified proteins from Additional file 1: Table S1.

\section{Abbreviations}

1-DE: One-dimensional gel electrophoresis; 2-DE: Two-dimensional gel electrophoresis; $\mathrm{Ab}$ : Antibody; $\mathrm{ddH}_{2} \mathrm{O}$ : double deionized water;

DIGE: Differential gel electrophoresis; DTT: Dithiothreitol; FCS: Fetal calf serum; MALDI-TOF MS: Matrix assisted laser desorption ionization-time of flight mass spectrometry. 


\section{Competing interests}

The authors declare that they have no competing interests.

\section{Authors' contributions}

HCC designed the experiments and wrote the drafting manuscript. JYC, RYH performed the cell culture, 2D-gel electrophoresis, image analysis, cell biological analysis and immunoblotting. HCC supervised the experiments and the data analysis and finalized the manuscript. All authors have read and approved the final manuscript.

Received: 11 September 2013 Accepted: 11 December 2013

Published: 20 December 2013

\section{References}

1. Verma S, Dent S, Chow BJ, Rayson D, Safra T: Metastatic breast cancer: the role of pegylated liposomal doxorubicin after conventional anthracyclines. Cancer Treat Rev 2008, 34:391-406.

2. Vatsyayan $R$, Chaudhary P, Lelsani PC, Singhal P, Awasthi YC, Awasthi S, Singhal SS: Role of RLIP76 in doxorubicin resistance in lung cancer (Review). Int J Oncol 2009, 34:1505-1511.

3. Green $A E$, Rose PG: Pegylated liposomal doxorubicin in ovarian cancer. Int J Nanomedicine 2006, 1:229-239.

4. Christiansen S, Autschbach R: Doxorubicin in experimental and clinical heart failure. Eur J Cardiothorac Surg 2006, 30:611-616.

5. Kalishina EV, Saprin AN, Solomka VS, Shchebrak NP, Piruzian LA: Inhibition of hydrogen peroxide, oxygen and semiquinone radicals in the development of drug resistance to doxorubicin in human erythroleukemia K562-cells. Vopr Onkol 2003, 49:294-298.

6. Simunek T, Sterba M, Popelova O, Adamcova M, Hrdina R, GersI V: Anthracyclineinduced cardiotoxicity: overview of studies examining the roles of oxidative stress and free cellular iron. Pharmacol Rep 2009, 61:154-171.

7. Joshi G, Aluise CD, Cole MP, Sultana R, Pierce WM, Vore M, St Clair DK, Butterfield DA: Alterations in brain antioxidant enzymes and redox proteomic identification of oxidized brain proteins induced by the anti-cancer drug adriamycin: implications for oxidative stress-mediated chemobrain. Neuroscience 2010, 166:796-807.

8. Keenan J, Murphy L, Henry M, Meleady P, Clynes M: Proteomic analysis of multidrug-resistance mechanisms in adriamycin-resistant variants of DLKP, a squamous lung cancer cell line. Proteomics 2009, 9:1556-1566.

9. Venkatakrishnan CD, Tewari AK, Moldovan L, Cardounel AJ, Zweier JL, Kuppusamy P, llangovan G: Heat shock protects cardiac cells from doxorubicin-induced toxicity by activating p38 MAPK and phosphorylation of small heat shock protein 27. Am J Physiol Heart Circ Physiol 2006, 291:H2680-H2691.

10. Boots AW, Haenen GR, Bast A: Health effects of quercetin: from antioxidant to nutraceutical. Eur J Pharmacol 2008, 585:325-337.

11. Sanhueza J, Valdes J, Campos R, Garrido A, Valenzuela A: Changes in the xanthine dehydrogenase/xanthine oxidase ratio in the rat kidney subjected to ischemia-reperfusion stress: preventive effect of some flavonoids. Res Commun Chem Pathol Pharmacol 1992, 78:211-218.

12. Sun B, Sun GB, Xiao J, Chen RC, Wang X, Wu Y, Cao L, Yang ZH, Sun XB: Isorhamnetin inhibits $\mathrm{H}$ (2) O (2)-induced activation of the intrinsic apoptotic pathway in $\mathrm{H} 9 \mathrm{c} 2$ cardiomyocytes through scavenging reactive oxygen species and ERK inactivation. J Cell Biochem 2012, 113:473-485.

13. Kyaw M, Yoshizumi M, Tsuchiya K, Kirima K, Tamaki T: Antioxidants inhibit JNK and p38 MAPK activation but not ERK $1 / 2$ activation by angiotensin II in rat aortic smooth muscle cells. Hypertens Res 2001, 24:251-261.

14. Staedler D, Idrizi E, Kenzaoui BH, Juillerat-Jeanneret L: Drug combinations with quercetin: doxorubicin plus quercetin in human breast cancer cells. Cancer Chemother Pharmacol 2011, 68:1161-1172.

15. Kaiserova H, Simunek T, van der Vijgh WJ, Bast A, Kvasnickova E: Flavonoids as protectors against doxorubicin cardiotoxicity: role of iron chelation, antioxidant activity and inhibition of carbonyl reductase. Biochim Biophys Acta 2007, 1772:1065-1074.

16. Zhou J, Liang S, Fang L, Chen L, Tang M, Xu Y, Fu A, Yang J, Wei Y: Quantitative proteomic analysis of HepG2 cells treated with quercetin suggests IQGAP1 involved in quercetin-induced regulation of cell proliferation and migration. OMICS 2009, 13:93-103.

17. Aalinkeel R, Bindukumar B, Reynolds JL, Sykes DE, Mahajan SD, Chadha KC, Schwartz SA: The dietary bioflavonoid, quercetin, selectively induces apoptosis of prostate cancer cells by down-regulating the expression of heat shock protein 90. Prostate 2008, 68:1773-1789.
18. Timms JF, Cramer R: Difference gel electrophoresis. Proteomics 2008, 8:4886-4897.

19. Lin ST, Chou HC, Chang SJ, Chen YW, Lyu PC, Wang WC, Chang MD, Chan HL: Proteomic analysis of proteins responsible for the development of doxorubicin resistance in human uterine cancer cells. J Proteomics 2012, 75:5822-5847.

20. Hung PH, Chen YW, Cheng KC, Chou HC, Lyu PC, Lu YC, Lee YR, Wu CT, Chan HL: Plasma proteomic analysis of the critical limb ischemia markers in diabetic patients with hemodialysis. Mol Biosyst 2011, 7:1990-1998.

21. Lin CP, Chen YW, Liu WH, Chou HC, Chang YP, Lin ST, Li JM, Jian SF, Lee YR, Chan HL: Proteomic identification of plasma biomarkers in uterine leiomyoma. Mol Biosyst 2011, 8:1136-1145.

22. Chen YW, Liu JY, Lin ST, Li JM, Huang SH, Chen JY, Wu JY, Kuo CC, Wu CL, $\mathrm{Lu} Y C$, et al: Proteomic analysis of gemcitabine-induced drug resistance in pancreatic cancer cells. Mol Biosyst 2011, 7:3065-3074.

23. Wu CL, Chou HC, Cheng CS, Li JM, Lin ST, Chen YW, Chan HL: Proteomic analysis of UVB-induced protein expression- and redox-dependent changes in skin fibroblasts using lysine- and cysteine-labeling two-dimensional difference gel electrophoresis. J Proteomics 2012, 75:1991-2014

24. Lai TC, Chou HC, Chen YW, Lee TR, Chan HT, Shen HH, Lee WT, Lin ST, Lu YC, $\mathrm{Wu} \mathrm{CL}$, et al: Secretomic and proteomic analysis of potential breast cancer markers by two-dimensional differential gel electrophoresis. J Proteome Res 2010, 9:1302-1322.

25. Chou HC, Lu YC, Cheng CS, Chen YW, Lyu PC, Lin CW, Timms JF, Chan HL: Proteomic and redox-proteomic analysis of berberine-induced cytotoxicity in breast cancer cells. J Proteomics 2012, 75:3158-3176.

26. Mokni M, Hamlaoui-Guesmi S, Amri M, Marzouki L, Limam F, Aouani E: Grape seed and skin extract protects against acute chemotherapy toxicity induced by doxorubicin in rat heart. Cardiovasc Toxicol 2012, 12:158-165.

27. Calvo-Romero JM, Fernandez-Soria-Pantoja R, Arrebola-Garcia JD, Gil-Cubero M: Ischemic heart disease associated with vincristine and doxorubicin chemotherapy. Ann Pharmacother 2001, 35:1403-1405.

28. Chen JY, Chan HL, Chou HC: Proteomic analysis of quercetin-induced cardioprotective effects. Genomic Med, Biomarkers, and Health Sci 2012, 4:51-53.

29. Lin ST, Chou HC, Chen YW, Chan HL: Redox-proteomic analysis of doxorubicin-induced altered thiol activity in cardiomyocytes. Mol Biosyst 2013, 9:447-456.

30. Chen YW, Chou HC, Lin ST, Chen YH, Chang YJ, Chen L, Chan HL: Cardioprotective effects of quercetin in cardiomyocyte under ischemia/ reperfusion injury. Evid Based Complement Alternat Med 2013, 2013:364519.

31. Oliva J, Bardag-Gorce F, Tillman B, French SW: Protective effect of quercetin, EGCG, catechin and betaine against oxidative stress induced by ethanol in vitro. Exp Mol Pathol 2011, 90:295-299.

32. Bongiovanni GA, Soria EA, Eynard AR: Effects of the plant flavonoids silymarin and quercetin on arsenite-induced oxidative stress in CHO-K1 cells. Food Chem Toxicol 2007, 45:971-976.

33. Gibellini L, Pinti M, Nasi M, De Biasi S, Roat E, Bertoncelli L, Cossarizza A: Interfering with ROS metabolism in cancer cells: the potential role of Quercetin. Cancers (Basel) 2010, 2:1288-1311.

34. Tytell M, Hooper PL: Heat shock proteins: new keys to the development of cytoprotective therapies. Expert Opin Ther Targets 2001, 5:267-287.

35. Takayama S, Krajewski S, Krajewska M, Kitada S, Zapata JM, Kochel K, Knee D, Scudiero D, Tudor G, Miller GJ, et al: Expression and location of Hsp70/ Hsc-binding anti-apoptotic protein BAG-1 and its variants in normal tissues and tumor cell lines. Cancer Res 1998, 58:3116-3131.

36. Zhang X, Xu Q, Saiki I: Quercetin inhibits the invasion and mobility of murine melanoma B16-BL6 cells through inducing apoptosis via decreasing Bcl-2 expression. Clin Exp Metastasis 2000, 18:415-421.

37. Dihal AA, der WH V, Hendriksen PJ, Charif H, Dekker LJ, ljsselstijn L, De Boer VC, Alink GM, Burgers PC, Rietjens IM, et al: Transcriptome and proteome profiling of colon mucosa from quercetin fed F344 rats point to tumor preventive mechanisms, increased mitochondrial fatty acid degradation and decreased glycolysis. Proteomics 2008, 8:45-61.

38. Shoshan V, Shahak Y, Shavit N: Quercetin interaction with the chloroplast ATPase complex. Biochim Biophys Acta 1980, 591:421-433.

doi:10.1186/1423-0127-20-95

Cite this article as: Chen et al:: Quercetin-induced cardioprotection against doxorubicin cytotoxicity. Journal of Biomedical Science 2013 20:95. 